Article

\title{
Characterization of Two VAO-Type Flavoprotein Oxidases from Myceliophthora thermophila
}

\author{
Alessandro R. Ferrari ${ }^{1}$, Henriëtte J. Rozeboom ${ }^{1}$, Aniek S. C. Vugts ${ }^{2}$, Martijn J. Koetsier ${ }^{2}$, \\ Robert Floor ${ }^{2}$ and Marco W. Fraaije ${ }^{1, * \text { (D) }}$ \\ 1 Molecular Enzymology, Groningen Biomolecular Sciences and Biotechnology Institute, \\ University of Groningen, 9747AG Groningen, The Netherlands; a.ferrari88@gmail.com (A.R.F.); \\ h.j.rozeboom@rug.nl (H.J.R.) \\ 2 Dupont Industrial Biosciences, 6709PA Wageningen, The Netherlands; aniek.vugts@dupont.com (A.S.C.V.); \\ martijn.koetsier@dupont.com (M.J.K.); robert.floor@dupont.com (R.F.) \\ * Correspondence: m.w.fraaije@rug.nl; Tel.: +31-503634345
}

Received: 8 December 2017; Accepted: 22 December 2017; Published: 5 January 2018

\begin{abstract}
The VAO flavoprotein family consists mostly of oxidoreductases harboring a covalently linked flavin cofactor. The linkage can be either monocovalent at position 8 with a histidine or tyrosine or bicovalent at position 8 with a histidine and at position 6 with a cysteine. Bicovalently bound flavoproteins show a preference for bulkier substrates such as oligosaccharides or secondary metabolites. The genome of the thermophilic fungus Myceliophthora thermophila $\mathrm{C} 1$ was found to be rich in genes encoding putative covalent VAO-type flavoproteins. Enzymes from this fungus have the advantage of being rather thermostable and homologous overexpression in $M$. thermophila $\mathrm{C} 1$ is feasible. Recently we discovered a new and VAO-type carbohydrate oxidase from this fungus: xylooligosaccharide oxidase. In this study, two other putative VAO-type oxidases, protein sequence XP_003663615 (MtVAO615) and XP_003665713 (MtVAO713), were expressed in M. thermophila C1, purified and characterized. Enzyme MtVAO615 was found to contain a bicovalently bound FAD, while enzyme MtVAO713 contained a monocovalent histidyl-bound FAD. The crystal structures of both proteins were obtained which revealed atypical active site architectures. It could be experimentally verified that both proteins, when reduced, rapidly react with molecular oxygen, a hallmark of flavoprotein oxidases. A large panel of alcohols, including carbohydrates, steroids and secondary alcohols were tested as potential substrates. For enzyme MtVAO713 low oxidase activity was discovered towards ricinoleic acid.
\end{abstract}

Keywords: oxidase; bicovalent; crystal structure; flavin adenine dinucleotide (FAD)

\section{Introduction}

Oxidases are enzymes capable of performing selective oxidative reactions using molecular oxygen as electron acceptor. They represent valuable and cost-effective biotechnological tools for industrial applications since they do not require expensive coenzymes but just molecular oxygen. To catalyze oxidations, oxidases typically employ a copper ion or a flavin as cofactor. Known examples of the former are galactose oxidase and laccases. The flavin-containing group of oxidases is more abundant. Oxidases in this category contain mostly a flavin adenine dinucleotide (FAD) as prosthetic group with a minority containing flavin mononucleotide (FMN). Based on sequence homology and the available structural information, six families of flavoprotein oxidases have been identified [1].

The FAD/FMN cofactor can be bound to the protein either non-covalently or covalently. The elucidation of the crystal structure of vanillyl alcohol oxidase (VAO) showed for the first time the FAD cofactor covalently bound to a protein via a histidine residue [2]. Members of the VAO flavoprotein family share a common overall structure which is composed of two domains: a conserved 
FAD binding domain that binds the adenine part of the FAD cofactor and a variable cap domain that covers the isoalloxazine ring of the cofactor and forms the substrate binding pocket [3]. In 2005 the crystal structure of another member of the VAO-type family, glucooligosaccharide oxidase (GOOX), was elucidated. This revealed for the first time a flavin cofactor covalently linked to two amino acids [4]. By having the FAD cofactor anchored by two covalent bonds, these bicovalent flavoproteins proteins seem to have evolved a rather open active site which allows them to accept bulky molecules as substrates [5]. It has also been shown that covalent tethering of the FAD to the protein increases the flavin redox potential, with bicovalent flavoproteins having the highest redox potentials [5]. With such high redox potentials, molecular oxygen is one of the few natural electron acceptors that covalent flavoproteins can use. This is probably the reason why most covalent VAO-type flavoproteins are oxidases [1].

Among the VAO-type oxidases, a great variety of substrates are accepted [6]. Some oxidases are part of gene clusters involved in tailoring secondary metabolites. Therefore they accept relatively complex biomolecules as substrates. Examples of these are Dbv29 that acts on the precursor of the glycopeptide A40926 [7], aclacinomycin oxidase which catalyzes the oxidation of a C-O bond and the dehydrogenation of the sugar moiety of aclacinomycin precursors [8], and the tirandamycin oxidase that catalyzes the oxidation of a secondary alcohol moiety of a tirandamycin intermediate [9]. Also the recently discovered subfamily of VAO-type carbohydrate oxidases, such as xylooligosaccharide oxidase (XylO) [10], chitooligosaccharide oxidase (ChitO) [11] or GOOX [12], act preferably on bulky oligosaccharides while monosaccharides are poorly recognized. The majority of VAO-type oxidases perform alcohol oxidations (EC 1.1.3.x) resulting in the production of aldehydes, ketones or lactones. Nonetheless, VAO, the prototype enzyme of this flavoprotein family, is not only able to perform alcohol oxidations but it is also capable to perform amine oxidations, hydroxylations and ether bond cleavage reactions [13]. Beside the aforementioned reactions, the VAO family includes also oxidases capable of C-C bound formation [14]. One known example is reticuline oxidase (also known as berberine bridge enzyme) involved in the biosynthetic pathway of plant isoquinoline alkaloids [15].

In an effort to discover novel oxidative biocatalysts acting towards biomass or biomass derived compounds, we specifically investigated the genome of the thermophilic fungus $M$. thermophila. This fungus is known to convert plant biomass and is a producer of a plethora of biomass converting enzymes. In addition, an effective $M$. thermophila expression system has been developed, enabling the production of high levels of recombinant proteins through homologous (and heterologous) expression. As M. thermophila is a thermophilic fungus, its proteins would withstand higher temperatures, making them optimal choices for industrial purposes.

We recently reported on the discovery and the elucidated crystal structure of a new VAO-type oxidase: xylooligosaccharide oxidase $(\mathrm{XylO})$ from $M$. thermophila $\mathrm{XylO}$ has a similar active site when compared to previously determined structures of other known VAO-type carbohydrate oxidases such as glucooligosaccharide oxidase from Acremonium strictum (GOOX) and lactose oxidase from Microdochium nivale $(\mathrm{LaO})$ [10]. Yet, for each of these enzymes, delicate differences in active site residues result in different substrate acceptance profiles. During the work that led to the discovery of $\mathrm{XylO}$, other putative VAO-type oxidases were identified in the predicted proteome of $M$. thermophila. In this study we report on the characterization of two of these VAO-type flavoproteins.

\section{Results}

\subsection{Identification of M. thermophila Genes Encoding Putative VAO-Type Flavoprotein Oxidases}

Using the sequence of the bicovalent flavoprotein ChitO as query, a BLAST search in the predicted proteome of $M$. thermophila was performed. Nineteen putative VAO-type flavoproteins were identified and subsequently aligned together with characterized VAO-type flavoproteins. Upon multiple sequence alignment of the recovered protein sequences with sequences from VAO-type flavoproteins for which a crystal structure was elucidated, a phylogenetic tree was built (Figure 1). Given their 
high similarity with MtVAO713, two additional sequences were included in the tree: an alcohol oxidase from Hypomyces subiculosus (accession number: ACD39759) and ecdysteroid-22-oxidase from Nomuraea rileyi (accession number: BAM11133). Thirteen clades can be identified in this phylogenetic tree. Eight clades mainly consist of known flavoproteins (clades I, III, IV, V, VI, VIII, XI, and XII). Clade V consists of the characterized carbohydrate oxidases, which includes $\mathrm{XylO}$, while also another homolog (XP_003661939.1) of M. thermophila is part of this group. This suggests that $M$. thermophila contains another carbohydrate oxidase. Other VAO-type proteins from M. thermophila are found in clades I, II, V, VII, IX, X, and XIII. Through SignalP (www.cbs.dtu.dk/services/ SignalP), eleven proteins of $M$. thermophila were predicted to contain a signal sequence for secretion: XylO, XP_003661939, XP_003663615, XP_003663619, XP_003662540, XP_003661824, XP_003663565, XP_003660778, XP_003665713, XP_003660023, XP_003659997. Interestingly the majority of these proteins are predicted to be bicovalently bound while only four (XP_003662540, XP_003665713, XP_003660023, XP_003659997) are predicted to be monocovalently bound. We selected two flavoproteins for further studies: XP_003663615 (MtVAO615), a putative bicovalent flavoprotein, and XP_003665713 (MtVAO713), a putative monocovalent flavoprotein. Interestingly, MtVAO713 has a Ser at the position where normally a Cys is present in bicovalent flavoproteins, forming the Cys-FAD linkage. Both proteins are part of a group of putative oxidases (clade II, see Figure 1) for which hardly any biochemical data are available and we set out to explore their catalytic and structural properties. 


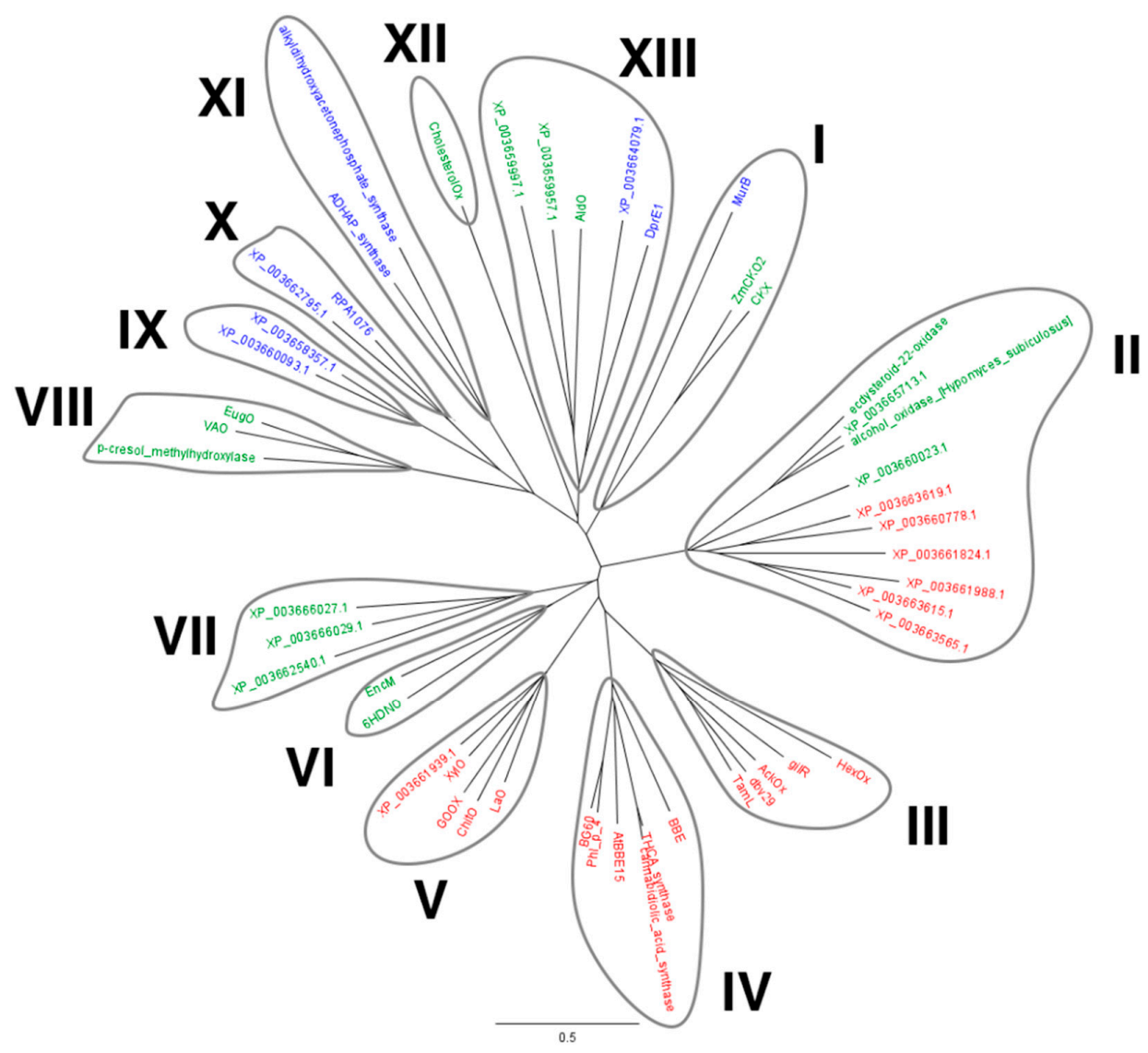

Figure 1. Phylogenetic tree of the VAO-type flavoproteins in the genome of $M$. thermophila supplemented with sequences of VAO-type proteins for which structures have been determined. Some covalent flavoproteins for which a structure is not available are also included: alcohol oxidase from H. subiculosus (ACD39759), ecdysteroid-22-oxidase from N. rileyi (BAM11133), and ChitO from Fusarium graminearum (XP_011325372). HexOx: hexose oxidase from Chondrus crispus (AAB49376), GilR: oxidoreductase from Streptomyces griseoflavus (Q7X2G7), AckOx: aclacinomycin oxidoreductase from Streptomyces galilaeus (ABI15166), dbv29: oxidase from Nonomuraea sp. ATCC 39727 (CAD91224), TamL: oxidase from Streptomyces sp. 307-9 (ADC79636), BBE: reticuline oxidase from Eschscholzia californica (P30986), THCA-synthase: $\Delta 1$-tetrahydrocannabinolic acid synthase from Cannabis sativa (Q8GTB6), cannabidiolic_acid_synthase: cannabidiolic acid synthase from C. sativa (AKC34419), Phl_p_4 from Phleum pretense (P43213), BG60 from Cynodon dactylon (AAS02108), LaO: carbohydrate oxidase from M. nivale (3RJA), GOOX from A. strictum (2AXR), XylO from M. thermophila (XP_003663758), MurB: MurB from Pseudomonas aeruginosa (4JAY), PCMH: p-cresol methylhydroxylase from Pseudomonas putida (P09788), VAO: vanillyl alcohol oxidase from Penicillium simplicissimum (P56216), EugO: eugenol oxidase from Rhodococcus jostii (5FXD), AtBBE15 from Arabidopsis thaliana (4UD8), HDNO: 6-hydroxy-D-nicotine oxidase from Arthrobacter oxydans (P08159), EncM: EncM from Streptomyces maritimus (Q9KHK2), CholesterolOx: cholesterol oxidase from Brevibacterium sterolicum (1I19), AldO: alditol oxidase from Streptomyces coelicolor (2VFR), DprE1: decaprenylphosphoryl-beta-D-ribose oxidase from M. tuberculosis (4FDN), ADHAP_synthase: alkyldihydroxyacetonephosphate synthase from Cavia porcellus (4BBY), alkyldihydroxyacetonephosphate synthase from Dictyostelium discoideum (2UUU), RPA1076: a putative dehydrogenase from Rhodopseudomonas palustris CGA009 (3PM9), ZmCKO2: cytokinin oxidase/dehydrogenase 2 from Zea mays (4ML8), CKX: cytokinin oxidase/dehydrogenase from Arabidopsis thaliana AT5G21482 (2EXR). The phylogenetic tree was generated with Geneious, version 7.0 (http:/ / www.geneious.com, Biomatters, Silkeborg, DK). In red: bicovalent flavoproteins. In green: monocovalent flavoproteins. In blue: non covalent flavoproteins. 


\subsection{Purification}

From $50 \mathrm{~mL}$ of concentrated fermentation supernatant, around $20 \mathrm{mg}$ of MtVAO615 was obtained after two purification steps. The purified protein displays a bright yellow color and exhibits and absorbance spectrum with maxima at 349 and $446 \mathrm{~nm}$ (Figure 2A). The spectral features (the $349 \mathrm{~nm}$ peak is rather broad and the $446 \mathrm{~nm}$ peak has a pronounced should at around $480 \mathrm{~nm}$ ) are reminiscent of those of bicovalent flavoproteins. The purified protein runs in SDS-PAGE as a single band at around $70 \mathrm{kDa}$ while the expected size is predicted to be $60 \mathrm{kDa}$. This indicates the presence of post-translational modifications such as glycosylation. Upon incubation of the gel with $5 \%$ acetic acid, the protein displayed clear fluorescence, confirming the presence of covalently bound FAD.

A

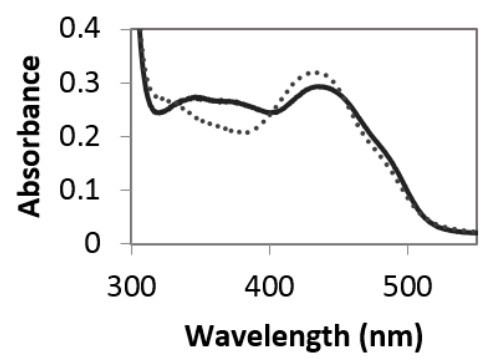

B

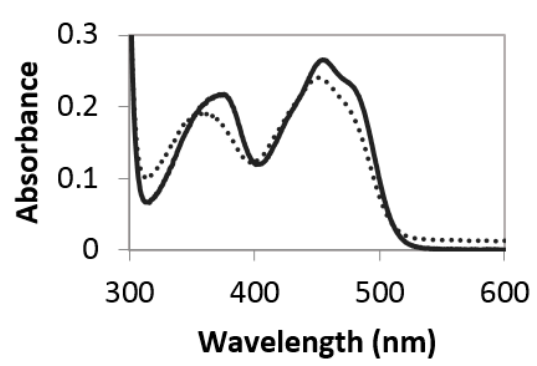

Figure 2. (A) Spectrum of $26 \mu \mathrm{M}$ native MtVAO615 (solid line) and unfolded MtVAO615 upon addition

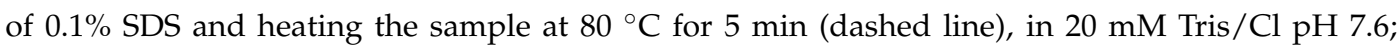
(B) Spectrum of $20 \mu \mathrm{M}$ native MtVAO713 (solid line) and unfolded MtVAO713 upon addition of $0.1 \%$ SDS and heating the sample at $80{ }^{\circ} \mathrm{C}$ for $5 \mathrm{~min}$ (dashed line), in $20 \mathrm{mM}$ Tris $/ \mathrm{Cl} \mathrm{pH} \mathrm{7.6.}$

From $50 \mathrm{~mL}$ of concentrated fermentation supernatant, around $45 \mathrm{mg}$ of MtVAO713 was obtained after two purification steps. The purified protein showed a bright yellow color and its absorbance spectrum displays maxima at 372 and $454 \mathrm{~nm}$ (Figure 2B). The absorbance spectrum is remarkably different from the one of MtVAO615 and hints to a non- or monocovalently bound flavin cofactor. On SDS-PAGE the purified fraction runs as a single band at around $60 \mathrm{kDa}$ which is the expected size for this protein. This suggests that this protein is not or not significantly glycosylated. Similar to MtVAO615, the protein displayed clear fluorescence upon SDS-PAGE and acetic acid treatment, indicating the presence of covalently bound FAD.

\subsection{Redox Potential Determination and Stopped Flow Experiments}

The redox potential of the FAD in both enzymes was investigated by using the xanthine oxidase-based method [16]. Using this method, a full reduction of both enzymes could be observed with no significant formation of a radical species. We were able to determine a redox potential of $+23 \mathrm{mV}$ for MtVAO713 using methylene blue $(+11 \mathrm{mV})$ as reference dye (Figure S2). Such a relatively high redox potential is typical for monocovalent flavoproteins. For MtVAO615 we were unable to calculate the redox potential since the redox potentials of the tested dyes were too far off from the one of the enzyme. In the redox titrations MtVAO615 became nearly fully reduced before thionin acetate $(+64 \mathrm{mV})$ was reduced (Figure S3) while with 2,6-dichloro indophenol $(+217 \mathrm{mV})$ it was the other way around. No other suitable reference dyes could be obtained. These observations indicate that the redox potential of MtVAO615 is extremely high for a flavoprotein, around $120-160 \mathrm{mV}$, which is close to the values reported for other bicovalent flavoproteins [5].

After having purified both proteins and having confirmed the presence of a covalent flavin cofactor, we set out to determine whether the proteins can function as oxidases. The typical characteristic of an oxidase is its capability to use molecular oxygen as electron acceptor. To test if the two purified proteins are oxidases, we first reduced anaerobically their flavin cofactor with 
sodium dithionite. Reduction could be witnessed by the disappearance of the yellow color of the oxidized flavin. After full reduction of the proteins, the proteins were mixed with a buffer containing dioxygen. By using a stopped-flow machine, the reappearance of oxidized flavin could be monitored in millisecond time scale. When mixing the reduced proteins with dioxygen, in both cases a rapid reoxidation of the reduced flavin was observed. Kinetic analysis of the spectral scans revealed that the reoxidation occurs in two (MtVAO713) or three (MtVAO615) steps (Figures S4 and S5). In both cases reappearance of the fully oxidized flavin spectrum mainly occurs in the first fast process with rates of $14.2 \pm 0.1 \mathrm{~s}^{-1}$ and $75.0 \pm 0.1 \mathrm{~s}^{-1}$, respectively. The subsequent kinetic events resulted in minor spectral changes which may reflect formation of flavin radical species or damaged flavin cofactor species, due to the intensity of the light source used for diode array detection. Therefore, they are probably irrelevant kinetic events. As a solution of dioxygen saturated buffer was used for reoxidation, the bimolecular rate constants could be calculated: $2.4 \times 10^{4} \mathrm{M}^{-1} \mathrm{~s}^{-1}$ for MtVAO713 and $1.3 \times 10^{5} \mathrm{M}^{-1} \mathrm{~s}^{-1}$ for MtVAO615. These rates are in the same range as those of known flavoprotein oxidases [17]. When taking into account that the rate of reoxidation is often even enhanced when product is still bound to the active site, these data show that both proteins are true flavoprotein oxidases.

\subsection{Substrate Screening}

Once the oxidase activity was confirmed for both flavoproteins, we set out to explore their substrate scope. First, a panel of twenty-three carbohydrates (see Materials and Methods was tested using the HRP-based assay. This assay couples the production of hydrogen peroxide, which is formed upon substrate oxidation by an oxidase, with the HRP-catalyzed formation of a purple product. Unfortunately, MtVAO615 nor MtVAO713 showed any activity towards the carbohydrates tested.

As can be seen in the phylogenetic tree (Figure 1), MtVAO713 is relatively closely related to an alcohol oxidase from $H$. subiculosus (GeneBank accession number: ACD39759.1; 59\% sequence identity) [18] and the ecdysteroid-22-oxidase from N. rileyi (GeneBank accession number: BAM11133.1; $52 \%$ sequence identity) [19]. Both fungal enzymes are acting on rather bulky secondary alcohols. Hinted by this, a plethora of steroids and secondary alcohols were tested by either the HRP assay or GC/MS in order to identify potential substrates. This revealed one substrate for MtVAO713: ricinoleic acid. Ricinoleic acid is the major component of the castor oil which is obtained from the pressing of the seeds of the plant Ricinus communis. It is an unsaturated omega-9 fatty acid hydroxylated in position 12. The oxidation in position 12 results in the formation of 12-ketooleic acid. Recently, a Korean group reported on the successful whole cell conversion of ricinoleic acid to 12-ketooleic acid by using a recombinant strain of Corynebacterium glutamicum expressing a secondary alcohol dehydrogenase from Micrococcus luteus [20]. The conversion of ricinoleic acid by MtVAO713 was confirmed by GC/MS analysis (Figures S6 and S7). Formation of 12-ketooleic acid could be measured. Nevertheless, the conversion rate is very poor as only $0.5 \%$ of $5 \mathrm{mM}$ ricinoleic acid was converted in $16 \mathrm{~h}$ by $8 \mu \mathrm{M}$ of MtVAO713. This indicates that ricinoleic acid is not the optimal substrate for this enzyme. Yet, it confirms that MtVAO713 can act as an alcohol oxidase and that it can convert secondary alcohols, a rare oxidase activity. Several ricinoleic acid derivatives were tested to unravel which functional groups are important for activity. None of the tested related compounds (methyl ricinoleate, ricinoleyl alcohol, 12-hydroxystearic acid, 12-hydroxydodecanoic acid) was found to be converted as measured by GC/MS.

For MtVAO615 a similar approach did not bring any lead. When examining the phylogenetic tree, no close homologs could be identified for which any biochemical data are available. A BLAST search results in several homologs with putative functions which have not been characterized yet. Several steroids, secondary alcohols and other different molecules were tested as potential substrate but no activity could so far be identified. Also, complex mixtures of substrates were tested as ground rye, oats, wheat, barley, wheat straw, arabinan and wheat arabinoxylan. These substrates would provide not only oligo- and polysaccharides but also other components of the plant material. MtVAO615 did not show activity with these complex mixtures. 


\subsection{Crystal Structures}

To have a better insight into the properties of both oxidases, their crystal structures were determined (Table 1). The structures of MtVAO615 and MtVAO713 were both determined by molecular replacement. The final model of MtVAO713 comprises 4 protein molecules with amino acid residues 27-598 and 4 FAD molecules. Electron density maps show the presence of $N$-glycosylation at Asn103 and Asn131. The final R-factors are 19.8/23.9 $\left(R_{\text {cryst }} / R_{\text {free }}\right)$. The crystal structure of MtVAO615 with amino acid residues 27-574 was solved in two different space groups. The structure at pH 7.5 contains one monomer while the structure at $\mathrm{pH} 5.0$ contains two monomers. Rcryst/Rfree are 19.5/23.5 and 23.6/28.0 respectively. As already observed by SDS-PAGE, MtVAO615 is heavily glycosylated, showing electron density in $2 \mathrm{~F}_{\mathrm{o}}-\mathrm{F}_{\mathrm{c}}$ maps for N-glycosylation at Asn47, Asn105, Asn129, Asn211, Asn310 and Asn438.

Table 1. Data collection and refinement statistics. Values in parentheses are for the highest resolution shell.

\begin{tabular}{|c|c|c|c|}
\hline & MtVAO615 & MtVAO615 & MtVAO713 \\
\hline Diffraction data & $\mathrm{pH} 7.5$ & pH 5.0 & \\
\hline Wavelength $(\AA)$ & 1.54 & 1.54 & 1.54 \\
\hline Resolution range $(\AA)$ & $51.4-2.0$ & $58.0-2.2$ & $54.3-2.2$ \\
\hline Spacegroup & $P 2{ }_{1} 2_{1} 2_{1}$ & $P 22_{1} 2_{1}$ & $P 2_{1}$ \\
\hline Cell dimensions $(\AA) a, b, c, \beta$ & $59.7,100.9,111.8$ & $62.4,116.0,198.7$ & $83.2,108.5,136.0,90.0$ \\
\hline Number of unique reflections & $45,720(2754)$ & $71,982(4265)$ & $120,924(5895)$ \\
\hline Completeness (\%) & $98.1(81.1)$ & $99.5(92.8)$ & $98.8(97.3)$ \\
\hline Overall I/ $\sigma(\mathrm{I})$ & $4.7(1.0)$ & $12.9(3.7)$ & $3.9(1.6)$ \\
\hline$R_{\text {merge }}(\%)$ & $17.2(98.6)$ & $14.7(51.4)$ & $16.1(48.3)$ \\
\hline$R_{\text {pim }}(\%)$ & $11.4(70.7)$ & $5.8(22.5)$ & $14.7(47.9)$ \\
\hline R/Rfree $(\%)$ & $23.6 / 28.0$ & $19.5 / 23.5$ & $19.8 / 23.9$ \\
\hline \multicolumn{4}{|l|}{ R.m.s. deviations from ideal values } \\
\hline Bond lengths $(\AA)$ & 0.010 & 0.009 & 0.013 \\
\hline Bond angles $\left({ }^{\circ}\right)$ & 1.444 & 1.333 & 1.575 \\
\hline Protein residues & $27-475$ & 27-475 (both molecules) & 30-598 (4 molecules) \\
\hline FAD molecule & 1 & $2 \times 1$ & $4 \times 1$ \\
\hline Water molecules & 366 & 850 & 1547 \\
\hline Glycerol molecules & - & & 8 \\
\hline NAG & 9 & $4(\mathrm{~A}), 7(\mathrm{~B})$ & $4 \times 3$ \\
\hline$\beta$-mannose & 1 & - & - \\
\hline PDB accession ID & $6 \mathrm{~F} 72$ & $6 \mathrm{~F} 73$ & $6 \mathrm{~F} 74$ \\
\hline
\end{tabular}

Both proteins belong to the VAO flavoprotein family. VAO-type proteins are composed of a FAD-binding domain and substrate domain [21]. MtVAO615 and MtVAO713 have 29\% sequence identity and have an rmsd of $2.0 \AA$ on $515 \mathrm{C} \alpha$ atoms. The structural differences are mainly situated in loops on the exterior of the proteins. Sequence alignment indicated that MtVAO615 and MtVAO713 contained an extra N-terminal domain of about 100 amino acid residues compared to other VAO flavoproteins. However, the extension is only $\sim 10$ residues. The remainder of the extra residues is found in 2 large loops in the FAD binding domain, several smaller loops and the C-terminal extension of $\sim 10$ amino acid residues (Figure S1). The first 26 residues of both proteins are part of secretion signal sequences. The MtVAO615 and MtVAO713 structures are similar to other structurally characterized VAO family members. The most similar structures to MtVAO615 are that of a flavoenzyme from Streptomyces maritimus (EncM, PDB code 3W8W, 22\% sequence identity rmsd $2.2 \AA$ ) [22], 6-hydroxy-D-nicotine oxidase from Arthrobacter nicotinovorans (6HDNO, PDB code 2BVF, 20\% sequence identity, rmsd $2.3 \AA$ [23]), aclacinomycin oxidoreductase from Streptomyces galilaeus (AckOx, PDB code 2IPI, 22\% sequence identity rmsd $2.4 \AA$ [8]), tirandamycin oxidase from Streptomyces sp. 307-9 (TamL, PDB code 2Y08, 22\% sequence identity, rmsd $2.3 \AA$ [9]) and lactose oxidase from Microdochium nivale (LaO, PDB code 3RJ8, 21\% sequence identity, rmsd $2.1 \AA$, to be published). For MtVAO713 these values are 21\%, $2.6 \AA$ (EncM); 20\%, $2.8 \AA$ (6HDNO); 15\%, $2.6 \AA$ (AckOx); 17\%, $2.4 \AA$ (TamL); and 20\%, $2.4 \AA$ (LaO). Structural similarity to MtVAO713 was highest for glucooligosaccharide oxidase from Acremonium strictum (GOOX, PDB code 1ZR6 19\% seq. id., rmsd 
$2.3 \AA$ [4]) and an oxidoreductase from Streptomyces griseoflavus (GilR, PDB code 3POP, $18 \%$ seq. id., rmsd $2.5 \AA$ [24]). The FAD domains of both structures are well conserved while conservation in the $S$ domains is much less, accounting for diverse substrate specificities (Figure S1).

Four disulfide bridges are present in MtVAO615 while in MtVAO713 six disulfide bridges exist. In both MtVAO615 and MtVAO713 the cofactor FAD is covalently tethered to the protein by covalent linkages. As expected, MtVAO615 harbors a bicovalently linked 6-S-cysteinyl-8 $\alpha$-N1-histidyl FAD while MtVAO713 has a monocovalently linked $8 \alpha$-methyl-N1-histidyl FAD (Figure S1). The isoalloxazine ring of the cofactor is covalently linked with the C6 atom to the S $\gamma$ of Cys222 and with the $8 \alpha$-methyl group to the N81 of His157 in MtVAO615 and His159 in MtVAO713. Cys222 in MtVAO615 is substituted for Ser231 in MtVAO713. All structural homologs have a covalently $8 \alpha$-methyl-N1-histidyl FAD while at the Cys222/Ser231 position, also Val or His residues are found (Figure S1).

The entrances to the active sites are shaped by the seven-stranded $\beta$-sheet of the substrate domain and $\alpha$-helix11 with a loop (res. 391-413 for MtVAO615, res. 415-433 for MtVAO713). The groove shaped substrate binding pocket of MtVAO615 is solvent accessible and contains, besides His157 and Cys222 which tether the FAD, Tyr159, Thr221, His237, Thr341, Tyr352, Leu399, cis-Pro401, Ala402, cis-Pro403, Ala408, Phe410, Thr413, Tyr449, Asp451, Leu475 Ala477, Ala479, Glu519 (Figure 3B). The structure of the loop is formed by two cis-prolines and is probably important for substrate binding. On the other side of the active site the disulfide Cys450-Cys476 stabilizes $\beta$-strands 17 and 18 with residues lining the active site. The groove to the substrate binding pocket of MtVAO713 is broader than of MtVAO615 and is delimited by Tyr100, Leu161, Ser231, His246, Tyr352, Phe354, Leu425, Asp427, Ile435, Ser433, Val469, Leu471, Ile501 and Glu543 (Figure 3A). The active site opposite to the FAD is very hydrophobic and is lined by several leucines and isoleucines. The hydrophobic nature of these residues is in line with the identified substrate ricinoleic acid, which is a very hydrophobic compound.

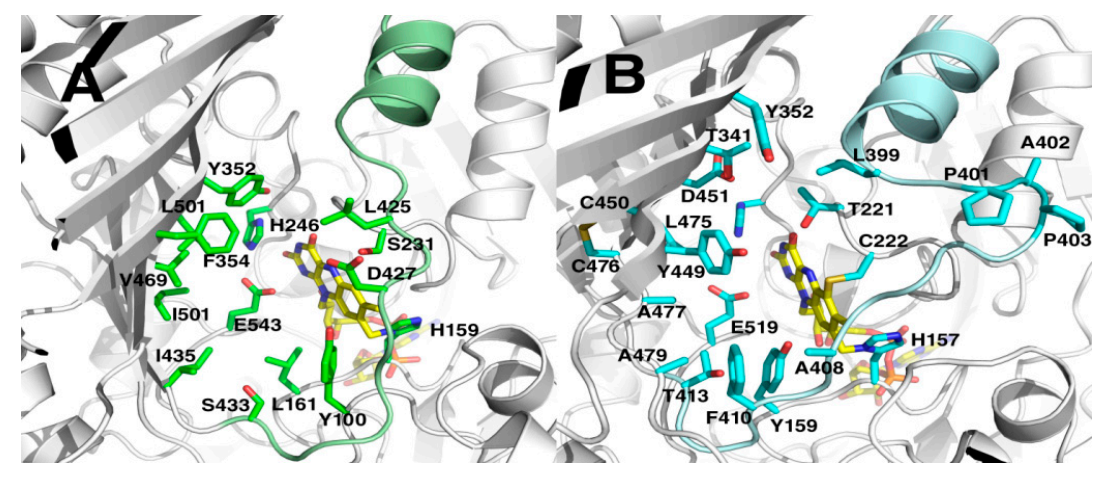

Figure 3. View of the predicted active sites of MtVAO713 (A) and MtVAO615 (B). The bound FAD (yellow) and active site pocket residues are in stick representations.

Among all the structurally characterized bicovalent flavoproteins, a common feature is the wide opening of the active site on the surface. This contrasts with monocovalent or noncovalent flavoproteins in which the active site is more buried. This feature allows bicovalent flavoproteins to accommodate bulky substrates in their active site [5]. For instance, carbohydrate oxidases in which the FAD is bicovalently bound accept oligosaccharides with higher efficiency compared to monosaccharides [3,11,12]. Protein MtVAO713 and MtVAO615 are both an exception to this rule. Despite having a monocovalent FAD, the active site of MtVAO713 is rather open, similar to bicovalent flavoproteins (Figure 3A). MtVAO713 is also closely related to other bicovalent flavoproteins concerning its sequence and structure. This suggests that MtVAO713 has evolved from a bicovalent flavoprotein.

On the other hand, despite having a bicovalently bound FAD cofactor, access to the active site of MtVAO615 is rather narrow (Figure 3B). In particular, Phe410 and Tyr449 seem to create a sort of funnel. It is worth to note that Phe410 is on a loop which runs longitudinally across the active site. While this 
loop is further away from the active site in the other sequence-related oxidases, in MtVAO615 it leans towards the active site contributing to narrowing the cavity. In physiological conditions and in the presence of the native substrate, the loop might be mobile to allow substrate binding.

When inspecting the active site residues of both MtVAO615 and MtVAO713, striking differences between these two proteins and the known structurally resolved oxidases can be noted. Table 2 lists the active site residues of various VAO-type flavoproteins for which a crystal structure is available. This shows that, while MtVAO615 and MtVAO713 have a strikingly similar active architecture, their active site residues are very different from other VAO-type oxidases. For example, a structurally conserved Tyr often assists in catalysis in most VAO-type oxidases which is missing in MtVAO615 and MtVAO713: a Glu residue is present at this position, respectively Glu519 and Glu543 (Table 2). It has been proposed that Tyr429 in GOOX acts as a base which abstracts a proton from the O1 of the substrate, contributing to the hydride transfer to FAD. A similar catalytic role is played by Tyr473 in Dbv29 [7], Tyr448 in GilR [24], Tyr447 in TamL [9], Tyr450 in aclacinomycin oxidoreductase (AckOx) [8] and Tyr484 in THCA synthase [25] (position '519' in Table 2). Asp355 in GOOX may assist in the proton transfer by lowering the pKa value of Tyr429 through a water molecule [4]. MtVAO615 has an Asp at a similar position, while MtVAO713 has a Leu (position 451 in Table 2). When comparing the active site residues, it seems that MtVAO615 and MtVAO713 represent a new subclass of oxidases with a totally new constellation of active site residues. While we could confirm oxidase activity on a secondary alcohol for MtVAO713, the real substrates for these oxidases still need to be identified. With the unique active sites, the oxidases may even catalyze unprecedented oxidative reactions as other sequence-related VAO-type oxidases have been shown to display various exotic oxidative reactions which include ether bond cleavage and $\mathrm{C}-\mathrm{C}$ bond formation reactions.

Inspection of the structures of both oxidases revealed a feature that is in line with the efficient oxygen reactivity. Recently a so-called gatekeeper residue which tunes oxygen reactivity of VAO-type flavoproteins was identified on the re-side of the isoalloxazine ring [26]. While this residue is typically rather bulky in homologs that are poorly reactive with dioxygen, in these newly identified oxidases residues (Val225 in MtVAO615 and Pro234 in MtVAO713) are found that provide enough space for dioxygen to reach and react with the reduced flavin cofactor. Taken together, the kinetic and structural data indicate that the two studied flavoproteins are bona vide oxidases.

Table 2. Amino acid positions relative to MtVAO615 in the active site of structurally charac-terized VAO-type flavoproteins (in grey hydrophobic residues, in red negatively charged residues, in blue positively charged residues, in green polar residues). Question marks indicate dubious residues given the lack of structural similarity with MtVAO615. 1DII: p-cresol methylhydroxylase from P. putida; 1VAO: VAO from P. simplicissimum; MtVAO713: XP_003665713.1 from M. thermophila; MtVAO615: XP_003663615.1 from M. thermophila; 3POP: GilR from S. griseoflavus; 2IPI: aclacinomycin oxidoreductase from S. galilaeus; 5AWV: Dbv29 from N. sp. ATCC 39727; 2Y08: TamL from S. sp. 307-9; 3D2D: reticuline oxidase from E. californica; 3VTE: $\triangle 1$-tetrahydrocannabinolic acid synthase from C. sativa; 4UD8: AtBBE15 from A. thaliana; 3TSH: Phlp4 from P. pretense; 4DNS: BG60 from C. dactylon; 3RJ8: lactose oxidase from M. nivale; 5L6F: XylO from M. thermophila; 2AXR: GOOX from A. strictum; ChitO from F. graminearum; 2BVF: 6-hydroxy-D-nicotine oxidase from A. oxydans; 3W8W: EncM from $S$. maritimus; 1I19: cholesterol oxidase from B. sterolicum; 2VFR: alditol oxidase from S. coelicolor; 4KW5: decaprenylphosphoryl-beta-D-ribose oxidase from M. tuberculosis; 4BBY: alkyldihydroxyacetonephosphate synthase from C. porcellus.

\begin{tabular}{ccccccccc}
\hline Position Relative to MtVAO615 & & $\mathbf{1 5 9}$ & & $\mathbf{2 3 7}$ & $\mathbf{4 5 1}$ & $\mathbf{4 7 5}$ & $\mathbf{4 7 7}$ & $\mathbf{5 1 9}$ \\
\hline 1DII & & $\mathrm{F}$ & & $\mathrm{V}$ & $\mathrm{I}$ & $\mathrm{H}$ & $\mathrm{V}$ & $\mathrm{Y}(?)$ \\
1VAO & $\mathrm{Y}$ & & $\mathrm{S}$ & $\mathrm{V}$ & $\mathrm{T}$ & $\mathrm{H}$ & $\mathrm{I}$ & $\mathrm{Y}(?)$ \\
\hline
\end{tabular}


Table 2. Cont.

\begin{tabular}{|c|c|c|c|c|c|c|}
\hline Position Relative to MtVAO615 & 159 & 237 & 451 & 475 & 477 & 519 \\
\hline 5FXD & G & $\mathrm{V}$ & I & $\mathrm{H}$ & V & $\mathrm{Y}(?)$ \\
\hline MtVAO713 & $\mathrm{L}$ & $\mathrm{H}$ & $\mathrm{L}$ & V & I & E \\
\hline MtVAO615 & $\mathrm{Y}$ & $\mathrm{H}$ & $\mathrm{D}$ & $\mathrm{L}$ & A & $\mathrm{E}$ \\
\hline 3POP & G & $\mathrm{Y}$ & $\mathrm{N}$ & K & $\mathrm{S}$ & $\mathrm{Y}$ \\
\hline 2IPI & $\mathrm{F}$ & $\mathrm{Y}$ & $\mathrm{Y}$ & K & W & Y \\
\hline $5 \mathrm{AWV}$ & F & Y & I & K & $\mathrm{N}$ & Y \\
\hline $2 Y 08$ & Y & $\mathrm{Y}$ & I & $\mathrm{K}$ & V & $\mathrm{Y}$ \\
\hline 3D2D & Y & F & $\mathrm{N}$ & M & E & $\mathrm{H}$ \\
\hline 3VTE & A & Y & $\mathrm{Y}$ & $\mathrm{E}$ & W & $\mathrm{Y}$ \\
\hline 4UD8 & Y & Y & $\mathrm{N}$ & K & $Q$ & Y \\
\hline $3 \mathrm{TSH}$ & Y & F & D & $\mathrm{N}$ & $\mathrm{Q}$ & Y \\
\hline 4DNS & Y & $\mathrm{F}$ & D & $\mathrm{N}$ & $\mathrm{Q}$ & $Y$ \\
\hline 3RJ8 & Y & F & $\mathrm{D}$ & $\mathrm{L}$ & $\hat{\mathrm{Q}}$ & Y \\
\hline $5 \mathrm{~L} 6 \mathrm{~F}$ & Y & F & $\mathrm{D}$ & $\mathrm{L}$ & $\mathrm{E}$ & Y \\
\hline $2 \mathrm{AXR}$ & Y & Y & D & $\mathrm{L}$ & Q & Y \\
\hline ChitO & Y & Y & D & $\mathrm{L}$ & Q & Y \\
\hline $2 \mathrm{BVF}$ & $\mathrm{P}$ & V & E & E & $\mathrm{L}$ & $\mathrm{N}$ \\
\hline $3 \mathrm{~W} 8 \mathrm{~W}$ & M & $\mathrm{F}$ & $\mathrm{L}$ & V & $\mathrm{N}$ & $\mathrm{F}$ \\
\hline $1 \mathrm{I} 19$ & W & $\mathrm{H}$ & $\mathrm{R}$ & W & $\mathrm{N}$ & $\mathrm{K}$ \\
\hline 2VFR & $\mathrm{F}$ & $\mathrm{H}$ & $\mathrm{R}$ & A & $\mathrm{H}$ & K \\
\hline 4KW5 & Y & $\mathrm{H}$ & $\mathrm{K}$ & $\mathrm{N}$ & C & $\mathrm{K}$ \\
\hline 4BBY & S & $\mathrm{H}$ & $\mathrm{T}$ & I & P & $\mathrm{H}(?)$ \\
\hline
\end{tabular}

\section{Discussion}

In the present study we report the discovery and the structural characterization of two novel flavoprotein oxidases from the thermophilic fungus $M$. thermophila. Both present rather unique structural features in the active site. Only for MtVAO713 we could identify ricinoleic acid as substrate (although a very poor one) which paves the way for further exploration.

The elucidation of the crystal structures can be used to rational design a library of compounds to be virtually screened through automated docking. Unfortunately this approach suffers of several limitations: (1) when lacking a lead compound, the creation of the library of test compounds might be extremely challenging and time consuming; (2) the results of a docking procedure often only predict whether binding of the compound is likely while it does not guarantee whether the binding pose will be productive, enabling catalysis; (3) the current docking procedures lacks accuracy in predicting binding energies which is partly due to the fact that typically the enzyme structure is taken as a rigid configuration.

Another approach for identifying a substrate for a newly found enzyme is testing complex mixtures of natural sources, for example extracts of plant biomass can be used to screen by chemical analysis for chemical modifications. This approach is less specific but in this lies its advantage when no indication whatsoever is available as to which substrate would work with these novel enzymes. In particular for MtVAO713, extracts can be generated from flax and/or oats hulls and be tested with the enzyme. The analysis will present definitely challenges given the high heterogeneity of the sample. Pre-fractionation of the extract could be used to reduce the sample complexity. Alternatively, it may be tempting to use protein crystals to soak them in a (complex) mixture of test compounds. When this is performed anaerobically, substrate will bind and by structure elucidation, the identity of tight binding substrates will be revealed.

\section{Materials and Methods}

\subsection{Bioinformatic Analysis and Expression}

The sequence of ChitO (accession number: XP_011325372) was used as query to perform a protein BLAST search on the "Non-redundant protein sequences" database with M. thermophila ATCC 42464 (taxid: 573729) set as organism (https:/ /blast.ncbi.nlm.nih.gov/Blast.cgi). Protein sequences were exported in the FASTA format and aligned with the software Geneious using default parameters. From the multiple sequence alignment, a phylogenetic tree was obtained with the following parameters: 
Genetic distance model: Jukes Cantor; Tree build method: Neighbor-Joining; Outgroup: No outgroup. Cloning and expression of MtVAO713 and MtVAO615 were performed as described in [10].

\subsection{Purification}

Purification was performed similar to a procedure of a previous study [10]. $50 \mathrm{~mL}$ processed fermentation broths for each protein were received from Dupont frozen at $-20{ }^{\circ} \mathrm{C}$. Samples were thawed on ice and buffer exchanged with $20 \mathrm{mM}$ Tris/ $\mathrm{Cl}$ pH 7.6. Subsequently they were loaded on pre-equilibrated $5 \mathrm{~mL}$ Hi-Trap Q-sepharose column using a FPLC purification system (ÅKTA Purifier) (GE Healthcare Europe GmbH, Eindhoven, NL). Absorbance was monitored at $280 \mathrm{~nm}, 445 \mathrm{~nm}$ and $600 \mathrm{~nm}$. The column was washed until absorbance at $280 \mathrm{~nm}$ reached baseline levels ( $\approx 10$ column volumes). Both proteins were eluted at $10 \%$ of elution buffer $(20 \mathrm{mM}$ Tris $/ \mathrm{Cl} \mathrm{pH} 7.6$ and $2 \mathrm{M} \mathrm{NaCl})$.

Yellow fractions which displayed absorbance at $445 \mathrm{~nm}$ were collected, concentrated and subsequently loaded on a Superdex 200 column. $50 \mathrm{mM}$ acetate buffer $\mathrm{pH} 5.6$ containing $100 \mathrm{mM} \mathrm{NaCl}$ was used to equilibrate the column and for the elution. The yellow fractions displaying absorbance at $445 \mathrm{~nm}$ were collected and concentrated.

\subsection{Crystallization, Data Collection and Structure Determination of MtVAO615}

Initial vapour-diffusion crystallization experiments were performed using a Mosquito crystallization robot (TTP Labtech, Hertfordshire, UK). In a typical experiment, $0.1 \mu \mathrm{L}$ screening solution was added to $0.1 \mu \mathrm{L}$ MtVAO615 protein solution $\left(9.6 \mathrm{mg} \mathrm{mL}^{-1}\right)$ on a 96 -well MRC2 plate (Molecular Dimensions, Suffolk, UK); reservoir wells contained $50 \mu \mathrm{L}$ screening solution. The screening solutions used for the experiments were PACT and JCSG+ (Molecular Dimensions). MtVAO615 crystals appeared after 3-30 d of incubation at $294 \mathrm{~K}$ in solutions containing PEG at pH 5 to 9 . Crystallization conditions were optimized using hanging-drop set-ups containing $1 \mu \mathrm{L}$ protein solution and $1 \mu \mathrm{L}$ reservoir solution at $294 \mathrm{~K}$. Crystals grown from $11 \%$ PEG3350, 20\% glycerol and $0.1 \mathrm{M}$ Hepes $\mathrm{pH}$ 7.5 and from $20 \%$ PEG6000, $0.2 \mathrm{M} \mathrm{NaCl}$ and $0.1 \mathrm{M}$ sodium acetate at $\mathrm{pH} 5.0$ were used for crystal structure determination.

Before data collection, crystals were briefly soaked in a cryoprotectant solution, consisting of $20 \%$ glycerol, $25 \%$ PEG3350 and $0.1 \mathrm{M}$ Hepes $\mathrm{pH}$ 7.5. X-ray diffraction data to $2.0 \AA$ A resolution were collected from a single cryo-cooled crystal mounted on an in-house MarDTB Goniostat System using $\mathrm{Cu}-\mathrm{K} \alpha$ radiation from a Bruker MicrostarH rotating-anode generator equipped with HeliosMX mirrors. Intensity data were processed using iMosflm [27]. The diffraction patterns were anisotropic with a weak zone of decreased resolution $(2.7 \AA)$ resulting in poor statistics for the high resolution shell (Table 1). The crystals belong to spacegroup P212121 with one monomer of $54 \mathrm{kDa}$ in the asymmetric unit. The VM is $2.7 \AA^{3} / \mathrm{Da}$ [28] with a solvent content of $54 \%$. Crystals grown in one month at $\mathrm{pH}$ 5.0 were also cryocooled with $20 \%$ glycerol. They belonged to space group P22121 and diffracted to $2.2 \AA$ resolution. With two monomers in the asymmetric unit the solvent content is $57 \%$ and $\mathrm{V}_{\mathrm{M}}$ is $2.9 \AA^{3} / \mathrm{Da}$ [28]. Intensity data were processed with XDS [29]. Data collection statistics are listed in Table 1.

Five structures were selected with highest identity to the C-terminal part of MtVAO615 i.e., 6HDNO (PDB code: 2BVF) [23], AckOx (PDB code: 2IPI) [8], TamL (PDB code: 2Y08) [9], EncM (PDB code: 3 W8W) [22], and LaO, (PDB code: 3RJ8). No homology model could be obtained for the 100 $\mathrm{N}$-terminal amino acids because of lack of sequence homology. Using these five structures as templates, homology models were generated with the SCWRL server [30]. Molecular replacement was performed with the program Phaser [31] using an ensemble of the five homology models.

Although Phaser was able to find a clear solution for the $P 2{ }_{1} 2_{1} 2_{1}$ data with one monomer in the asymmetric unit, the calculated initial phases were not good enough to build the structure. This is probably caused by the low sequence identity and the anisotropically diffracting crystals. The phases calculated by Phaser for two monomers in the asymmetric unit in the $\mathrm{P} 22_{1} 2_{1}$ data were sufficient for 
automatic model building by the Phase and build program in the PHENIX suite [32]. The refined model was used in determining the structure in the $P 2{ }_{1}{ }_{2} 2_{1}$ space group by Phaser.

\subsection{Crystallization, Data Collection and Structure Determination of MtVAO713}

Before crystallization of MtVAO713, an extra step of enzyme purification (and buffer exchange) was performed using a Superdex200 10/300 GL column (GE Healthcare Europe GmbH, Eindhoven, $\mathrm{NL}$ ), equilibrated with $20 \mathrm{mM}$ Hepes buffer, pH 7.5, containing $150 \mathrm{mM} \mathrm{NaCl}$. MtVAO713 eluted at a molecular weight of $53 \mathrm{kDa}$. Only the first fractions of the MtVAO713 peak containing FAD were pooled and concentrated. Dynamic light scattering (DLS) analysis (DynaPro NanoStar, Wyatt technology, Santa Barbara, CA, USA) indicates that the protein has a hydrodynamic radius of $3.38 \mathrm{~nm}$, with an apparent molecular weight of $58 \mathrm{kDa}$ and $5 \%$ polydispersity.

Crystallization conditions were screened using the Mosquito crystallization robot with a MtVAO713 solution of $10-23 \mathrm{mg} \mathrm{mL}^{-1}$ (see above). Many commercially available screens were tried but only with the Morpheus screen (Molecular Dimensions) thin yellow plate-like crystals appeared after 1 month of incubation at $294 \mathrm{~K}$. Conditions were optimized using hanging-drop set-ups with 25\% PEG3350 and $0.1 \mathrm{M}$ Tris $\mathrm{pH}$ 8.5-9.0 as precipitant, and drops containing $1 \mu \mathrm{L}$ protein solution $\left(10 \mathrm{mg} \mathrm{mL}^{-1}\right)$ and $1 \mu \mathrm{L}$ reservoir solution.

Crystals were soaked in a cryoprotectant prepared by the addition of $20 \%(w / v)$ glycerol to the reservoir solution. X-ray diffraction data to $2.2 \AA$ resolution were collected from a single cryo-cooled crystal mounted on the in-house X-ray source. Intensity data were processed using XDS [29]. The crystals (apparently) belong to space group P22121 with $a=83.2 \AA, b=108.5 \AA$ and $c=136.0 \AA$ and all angles $90^{\circ}$. With two monomers of $58 \mathrm{kDa}$ in the asymmetric unit the VM is $2.4 \AA^{3} / \mathrm{Da}$ [28] with a solvent content of $48 \%$.

MtVAO713 has 24\% identity to MtVAO615 and this structure was used as a template for molecular replacement. The homology model was generated with the SCWRL server [30] and molecular replacement was performed with the program Phaser [31]. Phaser obtained a distinct solution for the first monomer but a solution for the second monomer could not be calculated. Analysis of the data revealed systematic absences for $(0, k, 0)$ with $\mathrm{k}$ odd and for $(0,0,1)$ with 1 odd. However, the 001 reflections only spike at intermediate resolution displaying $1=2 \mathrm{n}$ presence, breaking down on lower and higher resolution suggesting a lower symmetry space group. After processing the data in space group P21, with a $\beta$ angle of 90.00 , Phaser was able to find four molecules in the asymmetric unit. Molecules A/D and B/C are related by an NCS operator that is close to a perfect twofold crystallographic rotation having P222 pseudosymmetry. The phases were good enough to build a model automatically with the Phase and build program in the PHENIX suite [32]. Data collection statistics are listed in Table 1.

\subsection{Refinement}

REFMAC5 was used for refinement of the models [33] and Coot [34] was used for manual rebuilding and map inspection. In the last rounds of refinement TLS groups were used. The quality of the models was analyzed with MolProbity [35], secondary structure elements were assigned with DSSP [36] and Promals3D was used for structure alignment [37]. Figures were prepared with PyMOL (http://www/pymol.org) and ESPript [38]. Atomic coordinates and experimental structure factor amplitudes have been deposited in the Protein Data Bank (PDB) with PDB IDs 6F72, and 6F73 and 6F74 for monomeric, dimeric MtVAO615 and MtVAO713, respectively.

\subsection{Carbohydrates and Primary Alcohol Substrate Screening}

The screening was performed as previously described [39]. In brief, oxidase activity of the two enzymes was detected by coupling $\mathrm{H}_{2} \mathrm{O}_{2}$ production to the oxidation of 4-aminoantipyrine and 3,5-dichloro-2-hydroxybenzenesulfonic acid by horseradish peroxidase (HRP). The formation of the resulting pink/purple colored product can be followed at $515 \mathrm{~nm}\left(\varepsilon_{515}=26 \mathrm{mM}^{-1} \mathrm{~cm}^{-1}\right)$. The reaction mixture for the carbohydrates/primary alcohol substrate screening contained $50 \mathrm{mM}$ 
phosphate buffer, $\mathrm{pH}$ 7.6, $0.1 \mathrm{mM}$ 4-aminoantipyrine, 1.0 mM 3,5-dichloro-2-hydroxybenzenesulfonic acid, 4.0 units HRP and $1 \mu \mathrm{M}$ of either enzymes. A panel of different carbohydrates comprising monomeric, dimeric, tetrameric, polymeric, cyclooligosaccharides and a range of primary alcohols were tested (see below). Potential substrates were tested in duplicate using concentrations of $50 \mathrm{mM}$ and $5.0 \mathrm{mM}$ for mono- and disaccharides, and $1.0 \mathrm{mM}$ and $0.5 \mathrm{mM}$ for tetra- and cyclo-oligosaccharides. To exclude $\mathrm{pH}$ effect, xylobiose and cellobiose in $50 \mathrm{mM}$ and $5.0 \mathrm{mM}$ were tested in $\mathrm{pH}$ range from 4.0 to $\mathrm{pH} 9.0$ using the Britton\&Robinson buffer. For screening complex mixtures $2 \mu \mathrm{M}$ of either enzyme were used to detect oxidase activity with the HRP assay on complex mixtures of substrates. The assay was performed in $50 \mathrm{mM}$ acetate buffer $\mathrm{pH} 5.6$ on $50 \mathrm{mg} / \mathrm{mL}$ ground rye, oats, wheat, barley, wheat straw, arabinan and wheat arabinoxylan. Full list of tested compounds: for MtVAO713: glucose, galactose, fructose, mannose, xylose, arabinose, $N$-acetyl-D-glucosamine, sucrose, maltose, lactose, sorbitol, xylitol, cellobiose, cellotetraose, $\alpha$-cyclodextrin, $\beta$-cyclodextrin, arabinan, chitosan, chitin, starch, maltotetraose, glycerol, hydroxymethylfurfural, xylobiose, testosterone, cholesterol, beta-ecdysone, 20-hydroxyecdysone, ergosterol, cholecalciferol, beta-sitosterol, stigmasterol, ricinoleic acid, methly ricinoleate, ricinoleyl alcohol, pinellic acid, cyclohexanol, 2-propanol, 2-butanol, 2-pentanol, 2-heptanol, 1-amino-2-propanol, 3-pentanol, 3-heptanol, 3-octanol; for MtVAO615: glucose, galactose, fructose, mannose, xylose, arabinose, $N$-acetyl-D-glucosamine, sucrose, maltose, lactose, sorbitol, xylitol, cellobiose, cellotetraose, $\alpha$-cyclodextrin, $\beta$-cyclodextrin, arabinan, chitosan, chitin, starch, maltotetraose, glycerol, hydroxymethylfurfural, xylobiose, ethanol, benzyl alcohol, glucose-6-phosphate, galactose-1-phosphate, mannose-6-phosphate, fructose-6-phosphate, xylan, arabinan, arabinoxylan, rye whole grain flour, oats flour, rye refined flour, wheat flour, barley flakes, straw, $\mathrm{N}$-acetyl muramate.

\subsection{Steroids and Secondary Alcohol Substrate Screening}

$1 \mu \mathrm{M}$ of MtVAO713 was used to perform the conversion of either $1 \%$ or $5 \mathrm{mM}$ of a panel of steroids and secondary alcohols (Supplementary information). As buffer $50 \mathrm{mM}$ Tris/ $\mathrm{Cl} \mathrm{pH} 8.0$ and $10 \%$ DMSO as co-solvent were used. Reactions were performed overnight at $30^{\circ} \mathrm{C}$ or $37^{\circ} \mathrm{C}$ at $300 \mathrm{RPM}$ in a final volume of $1 \mathrm{~mL}$ in a $2 \mathrm{~mL}$ Eppendorf tube. Reactions were extracted twice with ethyl acetate and injected in the GC/MS. Ricinoleic acid and derived substrates i.e., methyl ricinoleate and ricinoleyl alcohol, were further processed for derivatization with the TMS reagent. Samples were first evaporated and then resuspended in $60 \mu \mathrm{L}$ of MTBE (methyl tert-butyl ether). Subsequently $60 \mu \mathrm{L}$ of $1 \% \mathrm{TMS}$ (trimethylsilyl) in BSTFA ( $N, O-b i s(t r i m e t h y l s i l y l)$ trifluoroacetamide) were added. This was followed by incubation at $75{ }^{\circ} \mathrm{C}$ for $30 \mathrm{~min}$ to allow the derivatization. The final samples were injected into GC/MS. The GC was performed on a HP1 column (dimethylpolysiloxan, $45 \mathrm{~m} \times 0.25 \mathrm{~mm} \times 0.25 \mu \mathrm{m}$ ) with a temperature gradient from 40 to $250{ }^{\circ} \mathrm{C}\left(5^{\circ} \mathrm{C} / \mathrm{min}\right)$ with a solvent cut-off of $6 \mathrm{~min}$ and a split ratio of 100. Ketooleic acid eluted at $36.9 \mathrm{~min}$ and ricinoleic acid at $37.2 \mathrm{~min}$.

\subsection{Stopped Flow Analysis}

The reoxidation of both enzymes was monitored by measuring the absorbance change of the FAD in presence of saturating $\mathrm{O}_{2}$ concentrations. A solution of approximately $40 \mu \mathrm{M}$ of either enzyme was prepared in $50 \mathrm{mM}$ phosphate buffer $\mathrm{pH} 7.6$ to a final volume of $1 \mathrm{~mL}$ into a glass tube. The tube was flushed with $\mathrm{N}_{2}$ for $10-15 \mathrm{~min}$. Then, sodium dithionite $(10 \mathrm{mM})$ was added until the enzyme solution became bleached. In another glass tube a solution of only buffer was flushed for 10-15 min with $\mathrm{O}_{2}$. These solutions were mixed 1 to 1 in an Applied Photophysics SX20 stopped-flow apparatus and re-oxidation was monitored for $1 \mathrm{~s}$ using a photo diode array (PDA) detector between 186 and $724 \mathrm{~nm}$. The spectral scans were fitted using equations for double or triple exponential decays.

\subsection{Redox Potential Determination}

The redox potentials were determined by using the method described by Massey [17]. Reactions were performed in $50 \mathrm{mM}$ potatassium phosphate buffer $\mathrm{pH} 7.0$ at $25^{\circ} \mathrm{C}$. A cuvette with MtVAO615 
$(10 \mu \mathrm{M})$ or MtVAO713 $(5 \mu \mathrm{M})$, xanthine $(400 \mu \mathrm{M})$, methyl viologen $(2-5 \mu \mathrm{M})$ and redox dye $(5-10 \mu \mathrm{M})$ was made anaerobically by flushing with $\mathrm{N}_{2}$ for $10-15 \mathrm{~min}$. Subsequently, 50-60 $\mu \mathrm{g}$ of xanthine oxidase was added anaerobically to the cuvette and spectra were collected every 2 min during the reaction using a Jasco V-650 spectrophotometer (JASCO, Cremella, IT). The redox potentials were calculated by plotting the $\log ([\mathrm{ox}] /[\mathrm{red}])$ of the protein versus $\log ([\mathrm{ox}] /[\mathrm{red}])$ of the redox dye according to Minnaert et al. [40]. As reference redox dyes we used methylene blue $\left(E_{M}=+11 \mathrm{mV}\right)$, thionin acetate $\left(\mathrm{E}_{\mathrm{M}}=+64 \mathrm{mV}\right)$ and 2,6-dichloro indophenol $\left(\mathrm{E}_{\mathrm{M}}=+217 \mathrm{mV}\right)$.

Supplementary Materials: Supplementary materials are available online.

Acknowledgments: A.R.F. acknowledges the Netherlands Organisation for Scientific Research (NWO) for providing funding for this research, in the framework of the TASC Technology Area BIOMASS.

Author Contributions: A.R.F., H.J.R., M.J.K., R.F. and M.W.F. conceived and designed the experiments; A.R.F., H.J.R. and A.S.C.V. performed the experiments; A.R.F., H.J.R., M.J.K., R.F. and M.W.F. analyzed the data; A.R.F., H.J.R. and M.W.F. wrote the paper.

Conflicts of Interest: The authors declare no conflict of interest.

\section{References}

1. Dijkman, W.P.; de Gonzalo, G.; Mattevi, A.; Fraaije, M.W. Flavoprotein oxidases: Classification and applications. Appl. Microbiol. Biotechnol. 2013, 97, 5177-5188. [CrossRef] [PubMed]

2. Fraaije, M.W.; Van Berkel, W.J.; Benen, J.A.; Visser, J.; Mattevi, A. A novel oxidoreductase family sharing a conserved FAD-binding domain. Trends Biochem. Sci. 1998, 23, 206-207. [CrossRef]

3. Xu, F.; Golightly, E.J.; Fuglsang, C.C.; Schneider, P.; Duke, K.R.; Lam, L.; Christensen, S.; Brown, K.M.; Jørgensen, C.T.; Brown, S.H. A novel carbohydrate: Acceptor oxidoreductase from Microdochium nivale. Eur. J. Biochem. 2001, 268, 1136-1142. [CrossRef] [PubMed]

4. Huang, C.-H.; Lai, W.-L.; Lee, M.-H.; Chen, C.-J.; Vasella, A.; Tsai, Y.-C.; Liaw, S.-H. Crystal structure of glucooligosaccharide oxidase from Acremonium strictum: A novel flavinylation of 6-S-cysteinyl, 8alpha-N1-histidyl FAD. J. Biol. Chem. 2005, 280, 38831-38838. [CrossRef] [PubMed]

5. Heuts, D.P.H.M.; Scrutton, N.S.; McIntire, W.S.; Fraaije, M.W. What's in a covalent bond? On the role and formation of covalently bound flavin cofactors. FEBS J. 2009, 276, 3405-3427. [CrossRef] [PubMed]

6. Ewing, T.A.; Fraaije, M.W.; Mattevi, A.; van Berkel, W.J.H. The VAO/PCMH flavoprotein family. Arch Biochem. Biophys. 2017, 632, 104-117. [CrossRef] [PubMed]

7. Li, Y.S.; Ho, J.Y.; Huang, C.C.; Lyu, S.Y.; Lee, C.Y.; Huang, Y.T.; Wu, C.J.; Chan, H.C.; Huang, C.J.; Hsu, N.S.; et al. A unique flavin mononucleotide-linked primary alcohol oxidase for glycopeptide A40926 maturation. J. Am. Chem. Soc. 2007, 129, 13384-13385. [CrossRef] [PubMed]

8. Alexeev, I.; Sultana, A.; Mäntsälä, P.; Niemi, J.; Schneider, G. Aclacinomycin oxidoreductase (AknOx) from the biosynthetic pathway of the antibiotic aclacinomycin is an unusual flavoenzyme with a dual active site. Proc. Natl. Acad. Sci. USA 2007, 104, 6170-6175. [CrossRef] [PubMed]

9. Carlson, J.C.; Li, S.; Gunatilleke, S.S.; Anzai, Y.; Burr, D.A.; Podust, L.M.; Sherman, D.H. Tirandamycin biosynthesis is mediated by co-dependent oxidative enzymes. Nat. Chem. 2011, 3, 628-633. [CrossRef] [PubMed]

10. Ferrari, A.R.; Rozeboom, H.J.; Dobruchowska, J.M.; Van Leeuwen, S.S.; Vugts, A.S.C.; Koetsier, M.J.; Visser, J.; Fraaije, M.W.; Hart, G. Discovery of a xylooligosaccharide oxidase from Myceliophthora thermophila C1. J. Biol. Chem. 2016, 291, 23709-23718. [CrossRef] [PubMed]

11. Heuts, D.P.H.M.; Janssen, D.B.; Fraaije, M.W. Changing the substrate specificity of a chitooligosaccharide oxidase from Fusarium graminearum by model-inspired site-directed mutagenesis. FEBS Lett. 2007, 581, 4905-4909. [CrossRef] [PubMed]

12. Lin, S.; Yang, T.; Inukai, T.; Yamasaki, M.; Tsai, Y. Purification and characterization of a novel glucooligosaccharide oxidase from Acremonium stricture T1. Biochim. Biophys. Acta (BBA) Protein Struct. Mol. Enzymol. 1991, 18, 41-47. [CrossRef]

13. Fraaije, M.W.; Veeger, C.; van Berkel, W.J. Substrate specificity of flavin-dependent vanillyl-alcohol oxidase from Penicillium simplicissimum. Evidence for the production of 4-hydroxycinnamyl alcohols from 4-allylphenols. Eur. J. Biochem. 1995, 234, 271-277. [CrossRef] [PubMed] 
14. Winter, R.T.; Fraaije, M.W. Applications of flavoprotein oxidases in organic synthesis-Novel reactivities that go beyond amine and alcohol oxidations. Curr. Org. Chem. 2012, 16, 2542-2550. [CrossRef]

15. Winkler, A.; Hartner, F.; Kutchan, T.M.; Glieder, A.; Macheroux, P. Biochemical evidence that berberine bridge enzyme belongs to a novel family of flavoproteins containing a bi-covalently attached FAD cofactor. J. Biol. Chem. 2006, 281, 21276-21285. [CrossRef] [PubMed]

16. Massey, V. A simple method for determination of redox potentials. In Flavins and Flavoproteins 1990; Curti, B., Ronchi, S., Zanetti, G., Eds.; John Wiley, Sons, Ltd.: Hoboken, NJ, USA, 1991; xxiii + 945pp.

17. Mattevi, A. To be or not to be an oxidase: Challenging the oxygen reactivity of flavoenzymes. Trends Biochem. Sci. 2006, 31, 276-283. [CrossRef] [PubMed]

18. Reeves, C.D.; Hu, Z.; Reid, R.; Kealey, J.T. Genes for the biosynthesis of the fungal polyketides hypothemycin from Hypomyces subiculosus and radicicol from Pochonia chlamydosporia. Appl. Environ. Microbiol. 2008, 74, 5121-5129. [CrossRef] [PubMed]

19. Kamimura, M.; Saito, H.; Niwa, R.; Niimi, T.; Toyoda, K.; Ueno, C.; Kanamori, Y.; Shimura, S.; Kiuchi, M. Fungal ecdysteroid-22-oxidase, a new tool for manipulating ecdysteroid signaling and insect development. J. Biol. Chem. 2012, 287, 16488-16498. [CrossRef] [PubMed]

20. Lee, B.-H.; Lee, S.-B.; Kim, H.-S.; Jeong, K.-J.; Park, J.-Y.; Park, K.-M.; Lee, J.-W. Whole cell bioconversion of ricinoleic acid to 12-ketooleic acid by recombinant Corynebacterium glutamicum-based biocatalyst. J. Microbiol. Biotechnol. 2015, 25, 452-458. [CrossRef] [PubMed]

21. Leferink, N.G.H.; Heuts, D.P.H.M.; Fraaije, M.W.; van Berkel, W.J.H. The growing VAO flavoprotein family. Arch. Biochem. Biophys. 2008, 474, 292-301. [CrossRef] [PubMed]

22. Teufel, R.; Miyanaga, A.; Michaudel, Q.; Stull, F.; Louie, G.; Noel, J.P.; Baran, P.S.; Palfey, B.; Moore, B.S. Flavin-mediated dual oxidation controls an enzymatic Favorskii-type rearrangement. Nature 2013, 503, 552-556. [CrossRef] [PubMed]

23. Koetter, J.W.A.; Schulz, G.E. Crystal structure of 6-hydroxy-D-nicotine oxidase from Arthrobacter nicotinovorans. J. Mol. Biol. 2005, 352, 418-428. [CrossRef] [PubMed]

24. Noinaj, N.; Bosserman, M.A.; Schickli, M.A.; Piszczek, G.; Kharel, M.K.; Pahari, P.; Buchanan, S.K.; Rohr, J. The crystal structure and mechanism of an unusual oxidoreductase, GilR, involved in gilvocarcin $\mathrm{V}$ biosynthesis. J. Biol. Chem. 2011, 286, 23533-23543. [CrossRef] [PubMed]

25. Shoyama, Y.; Tamada, T.; Kurihara, K.; Takeuchi, A.; Taura, F.; Arai, S.; Blaber, M.; Shoyama, Y.; Morimoto, S.; Kuroki, R. Structure and function of $\Delta 1$-tetrahydrocannabinolic acid (THCA) synthase, the enzyme controlling the psychoactivity of Cannabis sativa. J. Mol. Biol. 2012, 423, 96-105. [CrossRef] [PubMed]

26. Zafred, D.; Steiner, B.; Teufelberger, A.R.; Hromic, A.; Karplus, P.A.; Schofield, C.J.; Wallner, S.; Macheroux, P. Rationally engineered flavin-dependent oxidase reveals steric control of dioxygen reduction. FEBS J. 2015, 282, 3060-3074. [CrossRef] [PubMed]

27. Battye, T.G.G.; Kontogiannis, L.; Johnson, O.; Powell, H.R.; Leslie, A.G.W. iMOSFLM: A new graphical interface for diffraction-image processing with MOSFLM. Acta Crystallogr. D Biol. Crystallogr. 2011, 67, 271-281. [CrossRef] [PubMed]

28. Matthews, B.W. Solvent content of protein crystals. J. Mol. Biol. 1968, 33, 491-497. [CrossRef]

29. Kabsch, W. XDS. Acta Crystallogr. D Biol. Crystallogr. 2010, 66, 125-132. [CrossRef] [PubMed]

30. Canutescu, A.A.; Shelenkov, A.A.; Dunbrack, R.L. A graph-theory algorithm for rapid protein side-chain prediction. Protein Sci. 2003, 12, 2001-2014. [CrossRef] [PubMed]

31. McCoy, A.J. Solving structures of protein complexes by molecular replacement with Phaser. Acta Crystallogr. D Biol. Crystallogr. 2007, 63, 32-41. [CrossRef] [PubMed]

32. Adams, P.D.; Afonine, P.V.; Bunkóczi, G.; Chen, V.B.; Davis, I.W.; Echols, N.; Headd, J.J.; Hung, L.-W.; Kapral, G.J.; Grosse-Kunstleve, R.W.; et al. PHENIX: A comprehensive Python-based system for macromolecular structure solution. Acta Crystallogr. D Biol. Crystallogr. 2010, 66, 213-221. [CrossRef] [PubMed]

33. Murshudov, G.N.; Skubák, P.; Lebedev, A.A.; Pannu, N.S.; Steiner, R.A.; Nicholls, R.A.; Winn, M.D.; Long, F.; Vagin, A.A. REFMAC5 for the refinement of macromolecular crystal structures. Acta Crystallogr. D Biol. Crystallogr. 2011, 67, 355-367. [CrossRef] [PubMed]

34. Emsley, P.; Lohkamp, B.; Scott, W.G.; Cowtan, K. Features and development of Coot. Acta Crystallogr. D Biol. Crystallogr. 2010, 66, 486-501. [CrossRef] [PubMed] 
35. Chen, V.B.; Arendall, W.B.; Headd, J.J.; Keedy, D.A.; Immormino, R.M.; Kapral, G.J.; Murray, L.W.; Richardson, J.S.; Richardson, D.C. MolProbity: All-atom structure validation for macromolecular crystallography. Acta Crystallogr. D Biol. Crystallogr. 2010, 66, 12-21. [CrossRef] [PubMed]

36. Kabsch, W.; Sander, C. Dictionary of protein secondary structure: Pattern recognition of hydrogen-bonded and geometrical features. Biopolymers 1983, 22, 2577-2637. [CrossRef] [PubMed]

37. Pei, J.; Kim, B.-H.; Grishin, N.V. PROMALS3D: A tool for multiple protein sequence and structure alignments. Nucleic Acids Res. 2008, 36, 2295-2300. [CrossRef] [PubMed]

38. Gouet, P.; Courcelle, E.; Stuart, D.I.; Métoz, F. ESPript: Analysis of multiple sequence alignments in PostScript. Bioinformatics 1999, 15, 305-308. [CrossRef] [PubMed]

39. Ferrari, A.R.; Lee, M.; Fraaije, M.W. Expanding the substrate scope of chitooligosaccharide oxidase from Fusarium graminearum by structure-inspired mutagenesis. Biotechnol. Bioeng. 2015, 112, 1074-1080. [CrossRef] [PubMed]

40. Minnaert, K. Measurement of the equilibrium constant of the reaction between cytochrome c and cytochrome a. Biochim. Biophys. Acta 1965, 110, 42-56. [CrossRef]

Sample Availability: Samples of the compounds are not available from the authors. 